\title{
The SOX2OT/miR-194-5p axis regulates cell proliferation and mobility of gastric cancer through suppressing epithelial-mesenchymal transition
}

\author{
RUQIONG WEI, CAN DING, RAQUEL ALARCÒN RODRÌGUEZ and MARÌA DEL MAR REQUENA MULLOR
}

Department of Nursing, Physiotherapy and Medicine, Universidad de Almería, Almería 04120, Spain

Received February 27, 2018; Accepted August 30, 2018

DOI: $10.3892 / \mathrm{ol} .2018 .9433$

\begin{abstract}
Recent studies reported that long noncoding RNAs (LncRNAs) were involved in tumorigenesis of various human cancer types, including gastric cancer (GC) through targeting microRNAs (miRNAs/miRs). The present study investigated the biological functions of LncRNA SOX2 overlapping transcript (SOX2OT)/miR-194-5p axis and its underlying mechanism in the tumor progression of GC. The results showed that relative expression of LncRNA SOX2OT was highly upregulated while the expression of miR-194-5p was down-regulated in GC tissues and cell lines (MGC-803, SGC-7901, MKN-74). Knockdown of SOX2OT inhibited cell proliferation, invasion and migration of GC cells (MGC803, MKN-74) through reducing epithelial-mesenchymal transition (EMT). Moreover, miR-194-5p was predicted to be one of the targets of SOX2OT through bioinformatics analysis and was verified by luciferase reporter assay. miR-194-5p expression was negatively regulated by SOX2OT expression in GC cells and miR-194-5p inhibitor was found to counteract the inhibitory effects of SOX2OT short hairpin (sh)RNA on cell proliferation and mobility through enhancing EMT in GC cells. Taken together, the in vitro experiments revealed that knockdown of SOX2OT inhibited cell proliferation and mobility through suppressing EMT via targeting miR-194-5p in GC. In addition, results from in vivo experiments showed that knockdown of SOX2OT suppressed GC tumor growth and matrix metalloproteinase (MMP)-2 and MMP-9 expression through inhibiting EMT. Besides that, relative expression of miR-194-5p was increased in sh-SOX2OT
\end{abstract}

Correspondence to: Professor Ruqiong Wei, Department of Nursing, Physiotherapy and Medicine, Universidad de Almería, Carretera de Sacramento s/n, 1.04 Edificio de Ciencias de Salud, Despacho, Almería 04120, Spain

E-mail: weiruqiongspain@163.com

Abbreviations: LncRNAs, long noncoding RNAs; GC, gastric cancer; miRNAs, microRNAs; EMT, epithelial-mesenchymal transition; SOX2OT, SOX2 overlapping transcript; SD, standard deviation

Key words: SOX2OT, miR-194-5p, gastric cancer, epithelial-mesenchymal transition group compared with sh-NC group. In summary, our study elucidated that the SOX2OT/miR-194-5p axis participated in the tumor progression of GC through regulation of EMT both in vitro and in vivo. Hence, targeting the SOX2OT/miR-194-5p axis may aid in establishing novel strategies for therapy of GC.

\section{Introduction}

Gastric cancer (GC) is characterized as the most common type of the malignant gastric tumor and also one of the most deadly cancers around the world (1). Majority of GC patients are often diagnosed at advanced stages for the reason that clinical symptoms of GC are not obvious at early stage. The prognosis of advanced GC patients remains extremely low with the 5-year overall survival rate less than $30 \%$, largely due to the local and systemic metastasis of GC $(2,3)$. Therefore, getting a better understanding of the mechanism of GC tumorgenesis and metastasis can help to improve the therapeutic treatments for this disease.

Increasing evidence support that long noncoding RNA (LncRNA), which consists more than 200 nucleotides without protein-coding capacity, plays a pivotal role in the tumorigenesis of various types of cancers (4). Aberrant expression of lncRNAs was often observed in several kinds of tumors and lncRNAs are also involved in many cellular processes including cell proliferation, apoptosis, invasion and metastasis (5-7). SOX2 is a transcription factor that is essential for the maintenance of self-renewal and the pluripotency of undifferentiated embryonic stem cells $(8,9)$. LncRNA SOX2 overlapping transcript (SOX2OT) is mapped to the human chromosome 3q26.3 (Chr3q26.3) locus and is transcribed in the same orientation as SOX2 (10). Liu et al reported that enhanced expression of IncRNA SOX2OT promoted colorectal cancer cells proliferation and motility and was associated with the outcome of colorectal cancer patients (11). SOX2OT could also promote lung cancer cell proliferation and was a prognostic indicator of poor survival (12). As in GC, high level of SOX2OT was reported to contribute to malignant status and poor prognosis in GC (13). However, the underlying mechanism about the effect of IncRNA SOX2OT on the GC progression is still limited.

MicroRNAs (miRNAs/miRs) are a group of single-stranded RNAs with approximately 22 nucleotides. miRNAs often bind to 3'-untranslated regions (3'-UTRs) of their target mRNAs to regulate their expression (14). miR-194-5p was down-regulated 
in gallbladder cancer cells and overexpressed miR-194-5p promoted cells into S-phase and apoptosis, suggesting that miR-194-5p acted as a tumor suppressor in gallbladder cancer (15). Su's study reported that knockdown of SOX2OT inhibited the malignant biological behaviors of glioblastoma stem cells via up-regulating the expression of miR-194-5p and miR-122 (16). However, the interaction between SOX2OT and miR-194-5p in the progression of GC is still unclear.

In our present study, we observed that SOX2OT was highly expressed in GC tissues and cell lines. Knockdown of SOX2OT inhibited cell proliferation and mobility of GC cells through suppressing epithelial-mesenchymal-transition (EMT) via targeting miR-194-5p. Our results shed light on finding new therapeutic strategies for GC treatment.

\section{Materials and methods}

Tissue samples. $\mathrm{GC}$ tumor tissues $(\mathrm{n}=30)$ and the same number of adjacent histological healthy tissues were obtained from GC patients who underwent surgical treatment without preoperative radiotherapy and/or chemotherapy. Informed consent was obtained from all individual participants included in the study. The specimens were snap-frozen in liquid nitrogen and stored at $-80^{\circ} \mathrm{C}$ until use. The study was approved by the Medical Ethics Committee of Universidad de Almería (Almería, Spain).

Cell culture. GC cell lines (MGC-803, SGC-7901, MKN-74) and human normal gastric epithelium cell line (GES-1) were obtained from American Type Culture Collection (ATCC) and cultured in RPMI-1640 culture medium (Gibco; Thermo Fisher Scientific, Inc., Waltham, MA, USA) with $10 \%$ fetal bovine serum (FBS; Gibco; Thermo Fisher Scientific, Inc.) at $37^{\circ} \mathrm{C}$ in a humidified $5 \% \mathrm{CO}_{2}$ incubator.

Reverse transcription-quantitative polymerase chain reaction $(R T-q P C R)$. Total RNA was isolated from cells with TRIzol reagent (Thermo Fisher Scientific, Inc.). cDNA was generated using the M-MLV reverse transcriptase (Clontech, Palo Alto, CA, USA) and One-Step SYBR PrimeScript RT-PCR kit (Takara Bio, Inc., Otsu, Japan) was used to detect the expression of SOX2OT. TaqMan MicroRNA Reverse Transcription kit (Applied Biosystems; Thermo Fisher Scientific, Inc.) was used for the reverse transcription of miR-194-5p. The expression of miR-194-5p was detected using TaqMan Universal Master Mix II. The primers used were as follows: GAPDH: F: 5'-CGCTGAGTACGTCGTGGAGT-3' and R: 5'-CGTCAA AGGTGGAGGAGTGG-3'. SOX2OT: F: 5'-TGCTACAAGACA ACACCCTGA-3' and R: 5'-CCAAAGCCATAACCAGATT-3'. miR-194-5p: F: 5'-GCGGCGGTGTAACAGCAACTCC-3' and R: 5'-ATCCAGTGCAGGGTCCGAGG-3'. U6: F: 5'-GCTTCG GCACATATACTAAAAT-3' and R: 5'-CGCTTCACGAAT TTGCGTGTCAT-3'. The amplification protocol included an initial denaturation step at $95^{\circ} \mathrm{C}$ for $10 \mathrm{~min}$, followed by 40 cycles of $95^{\circ} \mathrm{C}$ for $15 \mathrm{sec}$ and $60^{\circ} \mathrm{C}$ for $60 \mathrm{sec}$. The expression levels were calculated using the $2^{-\Delta \Delta C q}$ method with GAPDH used for the normalization of the mRNA, and U6 for the miRNA (17).

Cell transfection. Short hairpin RNA (shRNA) against SOX2OT and their negative control (sh-NC), miR-194-5p mimic or inhibitor and their negative controls (mimic NC, inhibitor NC) were synthesized (GenePharma, Shanghai, China). GC cells were seeded into a 6-well plate to reach 70-80\% confluence for transfection. Transfections were conducted using Lipofectamine 2000 reagent (Thermo Fisher Scientific, Inc.) according to manufacturer's protocol. The transfection efficacy was analyzed with RT-qPCR.

Cell proliferation assay. Cell proliferation was measured through Cell Counting Kit-8 (CCK-8) assay. Cells were seeded in 96-well plates at a density of 3,000 cells per well in triplicate. Then, $10 \mu \mathrm{l}$ of CCK-8 (Beyotime Institute of Biotechnology, Jiangsu, China) was added to each well at different time points (0-5 days). Cells were incubated for $2 \mathrm{~h}$ at $37^{\circ} \mathrm{C}$ in dark and the absorbance was recorded at $450 \mathrm{~nm}$.

Cell migration and invasion assay. For cell migration and invasion assay, chambers of $6.5 \mathrm{~mm}$ in diameter with an $8-\mathrm{mm}$ pore size (Corning Incorporated, Corning, NY, USA) were used. Cells were resuspended in $100 \mu 1$ of serum-free medium at a density of $1 \times 10^{5}$ cell $/ \mathrm{ml}$ and seeded into the upper chamber (pre-coated with $50 \mathrm{ng} / \mu \mathrm{l}$ Matrigel solution for the cell invasion assay and non-coated for the cell migration assay). Then, $600 \mu \mathrm{l}$ of $10 \%$ FBS medium was placed in the lower chamber. After incubation at $37^{\circ} \mathrm{C}$ for $24 \mathrm{~h}$, the cells on the upper membrane surface were mechanically removed. Cells that migrated or invaded to the lower surface of the membrane were stained with $20 \%$ Giemsa. Five random fields were chosen to count and take photos under a microscope (Olympus Corporation, Tokyo, Japan).

Western blot assay. Cells were lysed using RIPA (Beyotime Institute of Biotechnology) buffer and the protein concentrations were analyzed by the BCA protein assay kit (Beyotime Institute of Biotechnology). The same amount of proteins were separated by $10 \%$ SDS-PAGE gel and then transferred into PVDF membranes (EMD Millipore, Billerica, MA, USA). Membranes were blocked by $5 \%$ skim milk for $1 \mathrm{~h}$ at room temperature and then incubated with primary antibodies as follows: anti-E-cadherin (sc-71007), anti-N-cadherin (sc-59987), anti-Vimentin (sc-80975), anti-MMP-2 (sc-13594), anti-MMP-9 (sc-13520) and anti-GAPDH (sc-32233) (at a dilution 1:1,000; Santa Cruz Biotechnology, Inc., Dallas, TX, USA) overnight at $4^{\circ} \mathrm{C}$. Membranes were then washed three times with TBST and incubated with horseradish peroxidase conjugated secondary antibody for $1 \mathrm{~h}$ at room temperature. Then the antibody-bound proteins were detected using the ECL system (Bio-Rad Laboratories, Inc., Hercules, CA, USA).

Bioinformatics prediction. To predict the target miRNAs of LncRNA SOX2OT, the following prediction algorithms were used: TargetScan (http://www.targetscan.org/vert_71/), PICTAR (http://www.pictar.org/) and miRDB (http://mirdb. org/miRDB/custom.html) target prediction algorithms.

Dual luciferase reporter assay. The sequence of SOX2OT was amplified by PCR and cloned into pmirGLO Dual-luciferase miRNA Target Expression Vectors along with its mutant sequence of miR-194-5p binding sites (GenePharma, Shanghai, China). Cells were seeded into 96-well plate and co-transfected with SOX2OT-WT or SOX2OT-MUT and miR-194-5p mimics using Lipofectamine 2000 (Thermo Fisher Scientific, Inc.). The 
Dual-Luciferase Reporter Assay System (Promega Corporation, Madison, WI, USA) was used for testing the luciferase activity.

In vivo animal study. For the in vivo study, the stably transfected cells (sh-SOX2OT or sh-NC transfected MKN-74 cells) were used. shRNA against SOX2OT was constructed in pGPU6/GFP/Neo vector and then transfected into MKN-74 cells to construct the stably transfected cells. The 4-week old BALB/c nude mice were supplied by Animal Center of Universidad de Almería. Experiments with mice were conducted strictly in accordance with a protocol approved by the Administrate Panel on Laboratory Animal Care of China Medical University and the animal experiments were approved by the Medical Ethics Committee of Universidad de Almería. Mice were divided randomly into 2 groups: i) sh-NC group and ii) sh-SOX2OT group with 5 in each group. Each mouse was subcutaneously injected with $5 \times 10^{5}$ transfected cells in the right flank area for subcutaneous implantation. Tumor volumes $\left(\mathrm{mm}^{3}\right)$ were measured every 5 days and calculated according to the following formula: tumor volumes $\left(\mathrm{mm}^{3}\right)=$ length $\mathrm{x}$ width ${ }^{2} / 2$. The mice were sacrificed after 25 days post injection and tumor tissues were harvested for further analysis.

Statistical analysis. All results were presented as mean \pm standard deviation (SD). All differences were analyzed by SPSS 19.0 statistical software (SPSS, Inc., Chicago, IL, USA) with the Student's t-test (two tailed) or one-way ANOVA with Student-Newman-Keuls post hoc test. $\mathrm{P}<0.05$ was considered to indicate a statistically significant difference.

\section{Results}

SOX2OT is increased while miR-194-5p is decreased in GC tissues and cell lines. Firstly, we detected relative expression of SOX2OT and miR-194-5p in GC tissues and cell lines respectively through RT-qPCR (Fig. 1). Relative expression of SOX2OT was found significantly up-regulated while the expression of miR-194-5p was found down-regulated in GC tissues compared with healthy tissues $\left({ }^{* * *} \mathrm{P}<0.001\right.$; Fig. 1A and $\left.\mathrm{C}\right)$. Besides that, we also found GC cell lines (MGC-803, SGC-7901, MKN-74) expressed higher SOX2OT and lower miR-194-5p compared with human normal gastric epithelium cell line GES-1 $\left({ }^{* * *} \mathrm{P}<0.001,{ }^{* *} \mathrm{P}<0.01\right.$; Fig. 1B and D). MKN-74 and MGC-803 cells were chosen for our following experiments for they had higher SOX2OT expression and lower miR-194-5p expression among these GC cell lines. Our data indicated that SOX2OT was up-regulated while miR-194-5p was down-regulated in GC tissues and cell lines.

Knockdown of SOX2OT suppresses cell proliferation and mobility of GC cells through inhibiting EMT. To investigate the role of SOX2OT in GC progression, sh-SOX2OT and sh-NC were transfected into MKN-74 and MGC-803 cells respectively (Fig. 2). Sh-SOX2OT transfection significantly reduced SOX2OT expression compared with sh-NC group and the transfection efficiency was measured by RT-qPCR ( ${ }^{* *} \mathrm{P}<0.01$; Fig. 2A and C). We observed that knockdown of SOX2OT inhibited cell proliferation markedly vs. sh-NC group in both MKN74 and MGC-803 cells ( ${ }^{*} \mathrm{P}<0.05,{ }^{* *} \mathrm{P}<0.01$; Fig. $2 \mathrm{~B}$ and D). Moreover, results from cell invasion and migration assay showed that sh-SOX2OT significantly hampered invasion and migration of MKN-74 and MGC-803 cells compared with sh-NC group $\left({ }^{* *} \mathrm{P}<0.01\right.$; Fig. 3). We also measured relative expression of EMT-related proteins and found that the expression of epithelial marker E-cadherin was increased while the expression of mesenchymal markers $\mathrm{N}$-cadherin and Vimentin was decreased in sh-SOX2OT group compared with sh-NC group $\left({ }^{* *} \mathrm{P}<0.01\right.$, ${ }^{* * * *} \mathrm{P}<0.001$; Fig. 4). These results suggested that knockdown of SOX2OT suppressed cell proliferation and mobility of GC cells through inhibiting EMT.

miR-194-5p is a target of SOX2OT and miR-194-5p inhibitor counteracts the effects of SOX2OT shRNA on GC progression. We then set to explore the relationship between SOX2OT and miR-194-5p. Through bioinformatics analysis, we found that miR-194-5p was a predicted target of SOX2OT (Fig. 5A). miR-194-5p mimic or inhibitor was transfected into MKN-74 cells to increase or decrease miR-194-5p expression $\left({ }^{* *} \mathrm{P}<0.01\right.$; Fig. 5B). In order to verify that miR-194-5p was a target of SOX2OT, luciferase reporter assay was performed. The results demonstrated the direct binding relationship between miR-194-5p and SOX2OT ( ${ }^{* *} \mathrm{P}<0.01$; Fig. 5C). Relative expression of miR-194-5p was notably up-regulated in sh-SOX2OT group, indicating that miR-194-5p expression was negatively regulated by SOX2OT ${ }^{* *} \mathrm{P}<0.01$; Fig. 5D). In addition, transfection with miR-194-5p inhibitor enhanced cell proliferation and mobility of GC cells compared with shRNA + inhibitor NC group, suggesting that miR-194-5p inhibitor abolished the inhibitory effects of SOX2OT shRNA on tumor progression of GC $\left({ }^{*} \mathrm{P}<0.05,{ }^{* *} \mathrm{P}<0.01,{ }^{\#} \mathrm{P}<0.05,{ }^{\# \#} \mathrm{P}<0.01,{ }^{\# \#} \mathrm{P}<0.001\right.$; Fig. 5E-G). Besides that, relative expression of E-cadherin was down-regulated while the expression of $\mathrm{N}$-cadherin and Vimentin was up-regulated strikingly in shRNA + miR-194-5p inhibitor group compared with shRNA + inhibitor NC group $\left({ }^{* *} \mathrm{P}<0.01,{ }^{\# \#} \mathrm{P}<0.01\right.$; Fig. $5 \mathrm{H}$ and I). Results above demonstrated that miR-194-5p was a target of SOX2OT and was negatively regulated by SOX2OT. miR-194-5p inhibitor counteracted the inhibitory effects of SOX2OT shRNA on tumor progression via enhancing EMT in GC.

SOX2OT/miR-194-5p axis suppresses GC tumor growth and metastasis through inhibition of EMT in vivo. Our in vitro experiments suggested that knockdown of SOX2OT inhibited cell proliferation and mobility through up-regulating miR-194-5p in GC cells. Then we further explored the effect of SOX2OT/miR-194-5p axis on GC tumor growth and metastasis in vivo. BALB/c nude mice were injected with sh-SOX2OT or sh-NC transfected MKN-74 cells to form animal models for in vivo researches. Our data showed that tumors in sh-SOX2OT group grew slower and also smaller than sh-NC group $\left({ }^{*} \mathrm{P}<0.05,{ }^{* *} \mathrm{P}<0.01\right.$; Fig. 6A). Relative expression of miR-194-5p was found higher in tumors of sh-SOX2OT group vs. sh-NC group $\left({ }^{* *} \mathrm{P}<0.01\right.$; Fig. $\left.6 \mathrm{~B}\right)$. We then detected relative expression of metastasis related proteins (MMP-2 and MMP-9) through western blot and observed that MMP-2 and MMP-9 expression both decreased remarkably in sh-SOX2OT group $\left({ }^{* *} \mathrm{P}<0.01\right.$; Fig. 6C and D). Besides that, relative protein level of E-cadherin was increased while the level of Vimentin was decreased in sh-SOX2OT group compared with sh-NC group $\left(^{* * *} \mathrm{P}<0.001\right.$, 

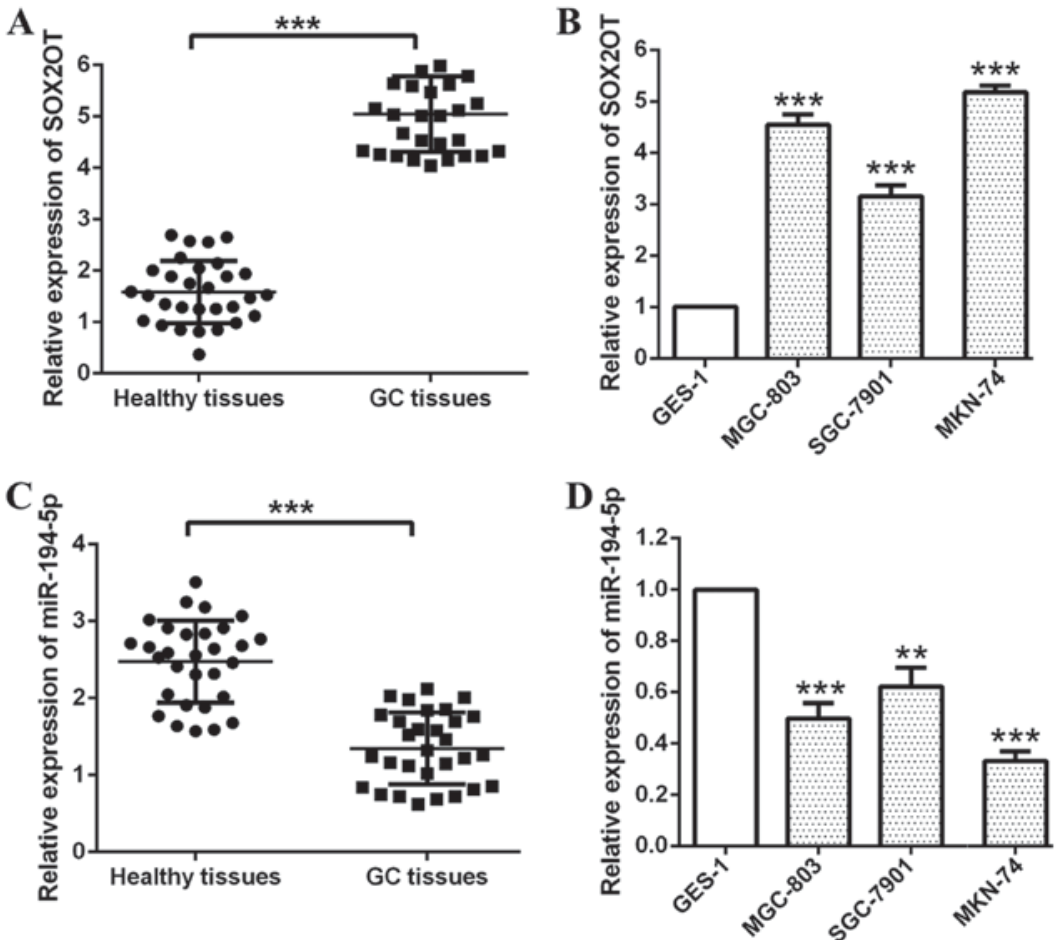

Figure 1. SOX2OT is increased while miR-194-5p is decreased in GC tissues and cell lines. Relative expression of SOX2OT in (A) GC tissues compared with healthy tissues and (B) GC cell lines compared with normal gastric cell line was detected by RT-qPCR. Relative expression of miR-194-5p in (C) GC tissues compared with healthy tissues and (D) GC cell lines compared with was detected by RT-qPCR. All data are presented as the mean \pm standard deviation from three independent experiments. ${ }^{* *} \mathrm{P}<0.01,{ }^{* * * *} \mathrm{P}<0.001$ compared with healthy tissues or GES-1 cells. SOX2OT, SOX2 overlapping transcript; miR, microRNA; GC, gastric cancer; RT-qPCR, reverse transcription-quantitative polymerase chain reaction.
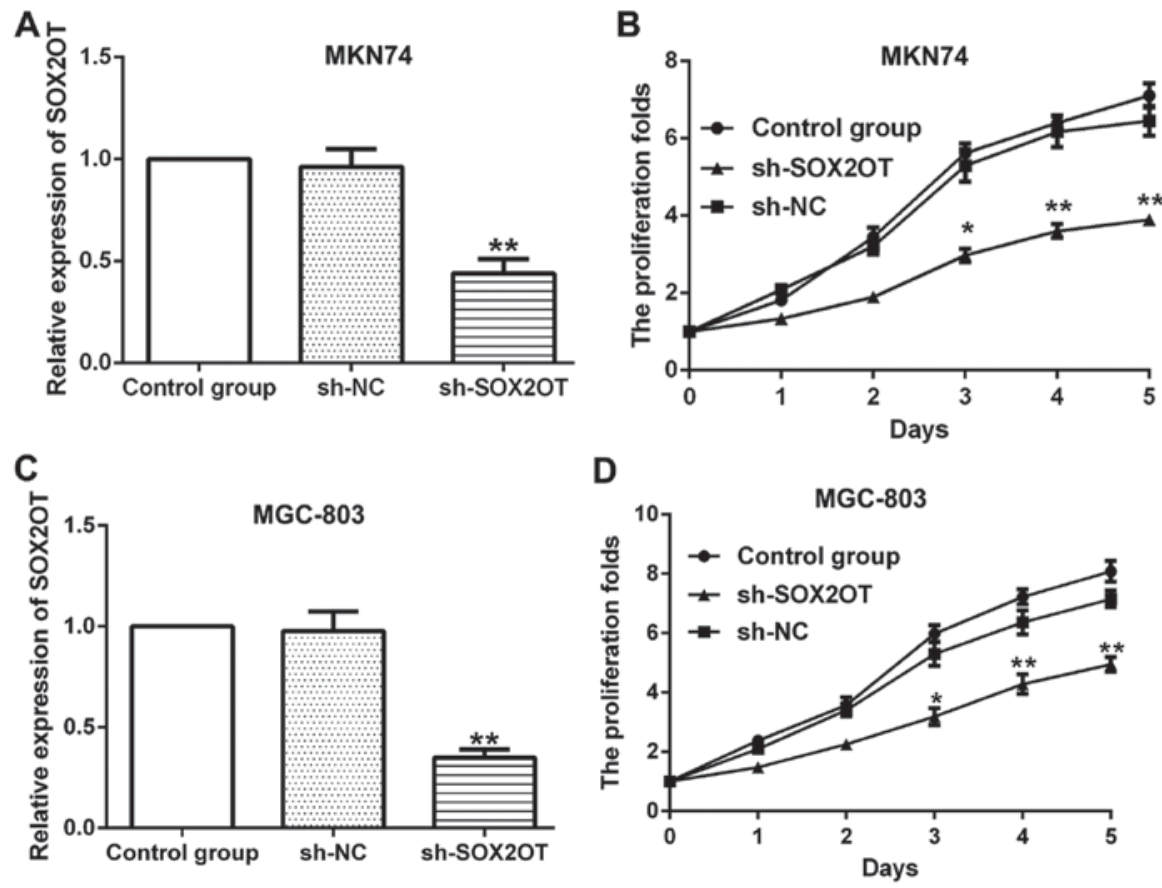

Figure 2. Knockdown of SOX2OT suppresses cell proliferation of GC cells. MKN-74 and MGC-803 cells were transfected with sh-SOX2OT or sh-NC respectively. (A) Relative expression of SOX2OT was detected through RT-qPCR and (B) cell proliferation was detected through CCK-8 assay in MKN-74 cells. (C) Relative expression of SOX2OT was detected through RT-qPCR and (D) cell proliferation was detected through CCK-8 assay in MGC-803 cells. All data are presented as the mean \pm standard deviation from three independent experiments. ${ }^{*} \mathrm{P}<0.05,{ }^{* * *} \mathrm{P}<0.01$ compared with sh-NC group. SOX2OT, SOX2 overlapping transcript; GC, gastric cancer; RT-qPCR, reverse transcription-quantitative polymerase chain reaction; CCK-8, Cell Counting Kit-8.

${ }^{* *} \mathrm{P}<0.01$; Fig. $6 \mathrm{E}$ and $\mathrm{F}$ ). Taken together, our in vivo experiments determined that SOX2OT/miR-194-5p axis suppressed
GC tumor growth and metastasis through inhibition of EMT in vivo. 
A

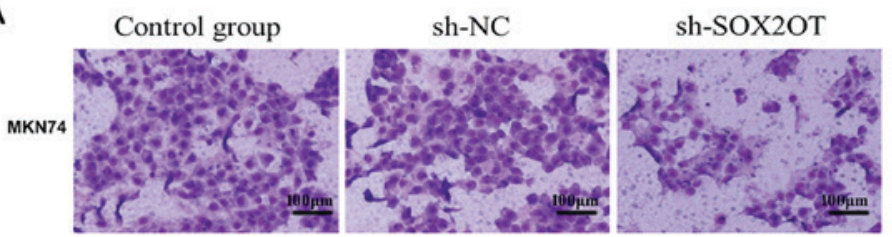

B

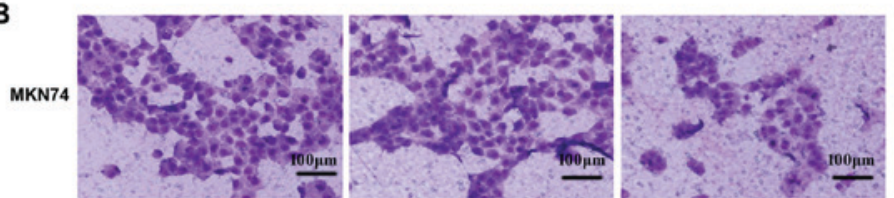

C
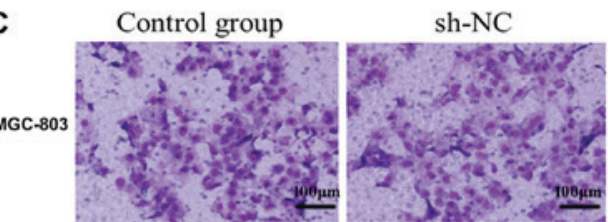

D

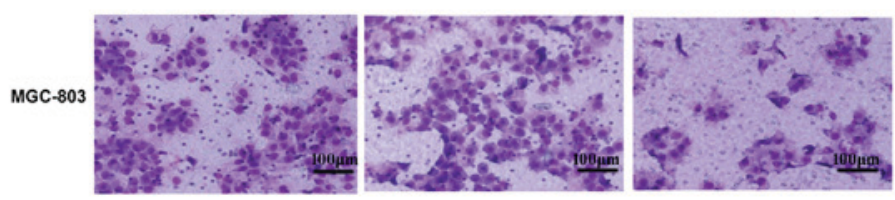

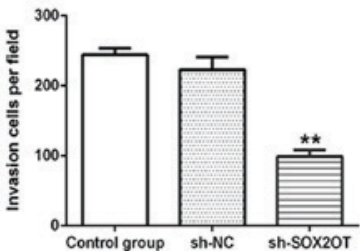

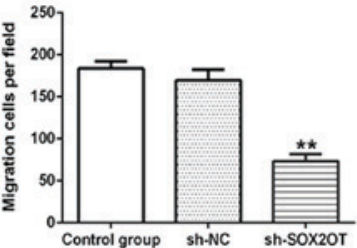

sh-SOX2OT
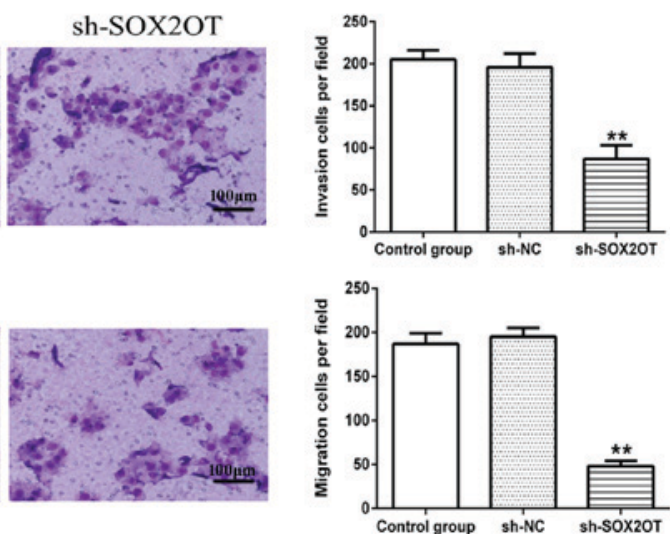

Figure 3. Knockdown of SOX2OT suppresses cell mobility of GC cells. MKN-74 and MGC-803 cells were transfected with sh-SOX2OT or sh-NC respectively. (A) Cell invasion and (B) cell migration abilities were detected using a Transwell model assay in MKN-74 cells. (C) Cell invasion and (D) cell migration abilities were detected using a Transwell model assay in MGC-803 cells. All data are presented as the mean \pm standard deviation from three independent experiments. ${ }^{* *} \mathrm{P}<0.01$ compared with sh-NC group. Scale bar, $100 \mu \mathrm{m}$. SOX2OT, SOX2 overlapping transcript; GC, gastric cancer.

A

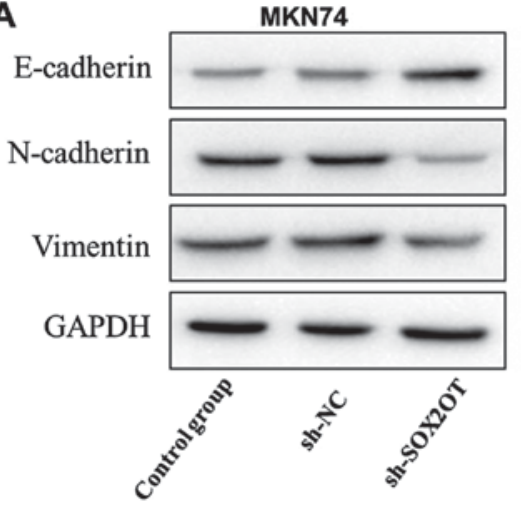

C

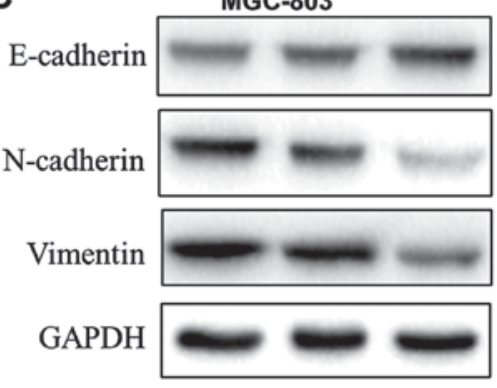

B

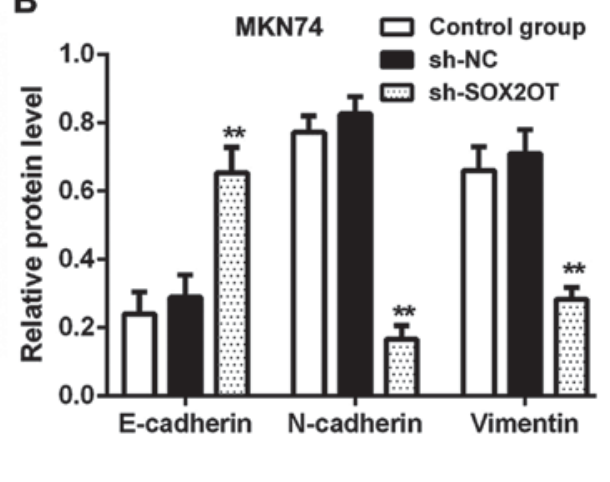

D

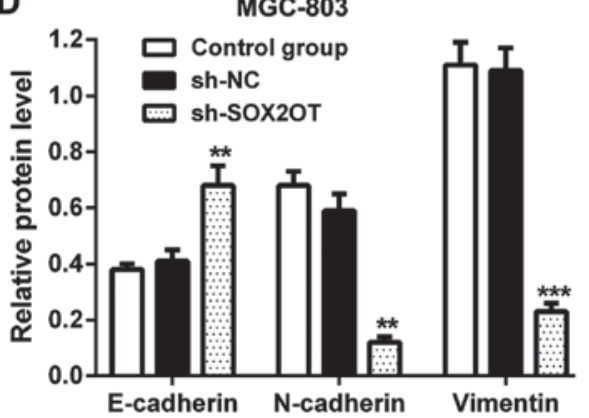

Figure 4. Knockdown of SOX2OT inhibits EMT in GC cells. MKN-74 and MGC-803 cells were transfected with sh-SOX2OT or sh-NC respectively, (A) Representative western blot and (B) quantification of E-cadherin, N-cadherin and Vimentin expression in MKN-74 cells. (C) Representative western blot and (D) quantification of E-cadherin, N-cadherin and Vimentin expression in MGC-803 cells. All data are presented as the mean \pm standard deviation from three independent experiments. ${ }^{* * *} \mathrm{P}<0.01,{ }^{* * *} \mathrm{P}<0.001$ compared with sh-NC group. SOX2OT, SOX2 overlapping transcript; EMT, epithelial-mesenchymal transition; GC, gastric cancer. 
A

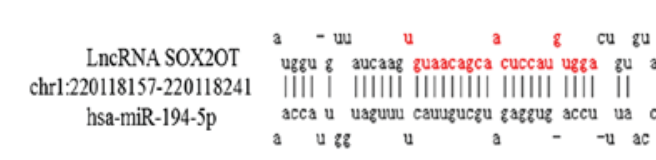

D

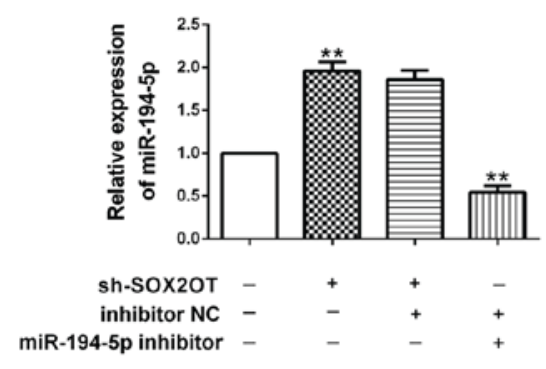

G

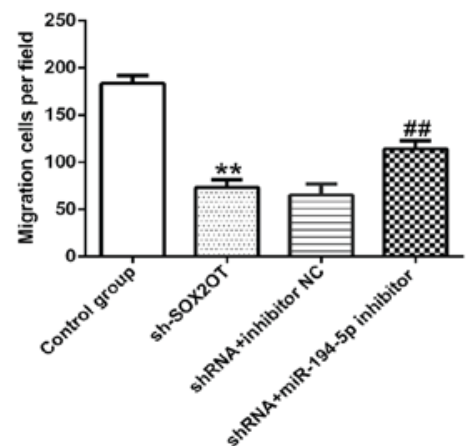

B

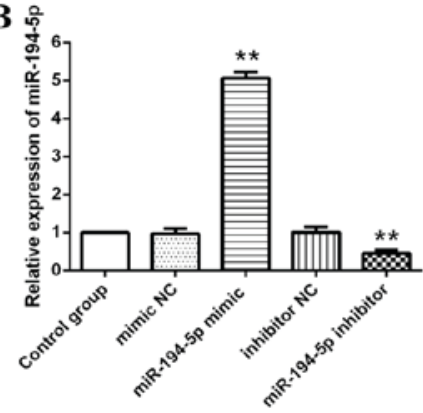

E

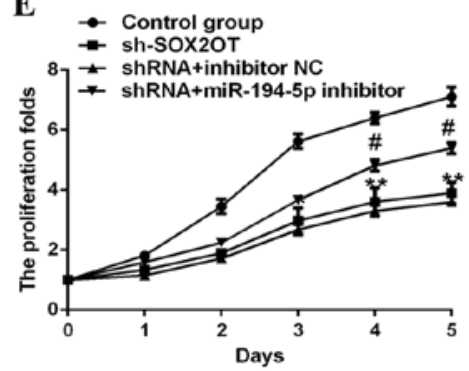

H

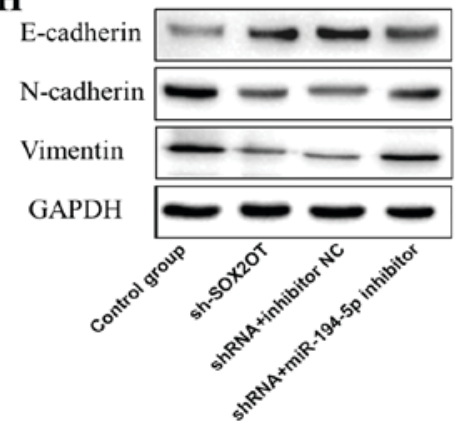

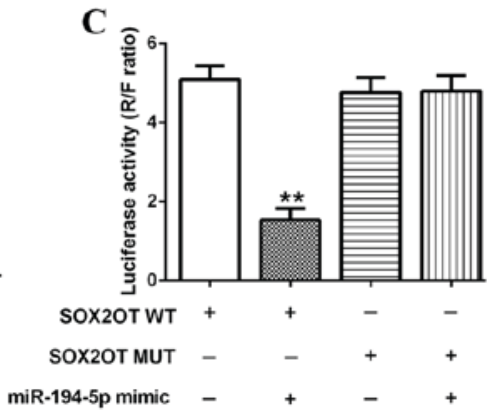

F

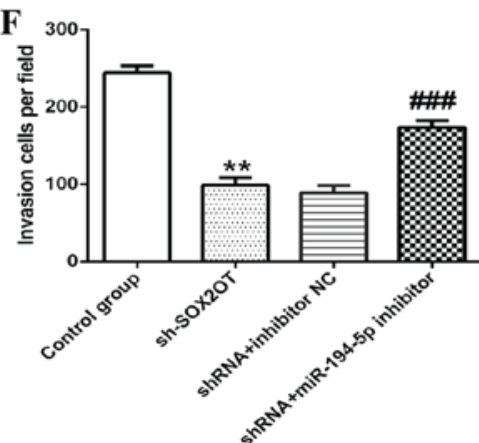

I

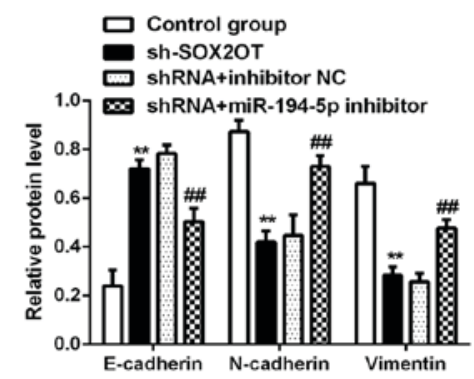

Figure 5. miR-194-5p is a target of SOX2OT and miR-194-5p inhibitor counteracts the effects of SOX2OT shRNA on GC progression. MKN-74 cells were transfected with sh-SOX2OT, sh-SOX2OT + inhibitor NC, sh-SOX2OT + miR-194-5p inhibitor respectively. (A) Target sequences of miR-194-5p in SOX2OT mRNA were analyzed through bioinformatics. (B) Relative expression of miR-194-5p was detected through RT-qPCR (** $<0.01$ compared with mimic NC or inhibitor NC group). (C) The interaction between SOX2OT and miR-194-5p was confirmed by luciferase reporter assay $\left(^{* *} \mathrm{P}<0.01\right.$ compared with SOX2OT WT group). (D) Relative expression of miR-194-5p was detected through RT-qPCR. (E) Cell proliferation was detected through CCK-8 assay. (F) Cell invasion and (G) migration abilities were detected using Transwell model assay. (H) Representative western blot and (I) quantification of E-cadherin, N-cadherin and Vimentin. All data are presented as the mean \pm standard deviation from three independent experiments. ${ }^{* *} \mathrm{P}<0.01$ compared with control group, ${ }^{*} \mathrm{P}<0.05$, ${ }^{\# \#} \mathrm{P}<0.01,{ }^{\# \#} \mathrm{P}<0.001$ compared with shRNA + inhibitor NC group. SOX2OT, SOX2 overlapping transcript; miR, microRNA; GC, gastric cancer; RT-qPCR, reverse transcription-quantitative polymerase chain reaction; CCK-8, Cell Counting Kit-8.

\section{Discussion}

GC is a common malignancy with high mortality rate. Patients with distant metastasis often miss the best time for operation and also have poor prognosis. Therefore, finding novel target to suppress GC cells proliferation and metastasis can be instrumental in improving the 5-year survival rates of GC patients. Recently, the participation of LncRNA in the tumor progression has gained much attention of researchers. In our present study, we indicated that LncRNA SOX2OT was up-regulated while miR-194-5p was down-regulated in GC tissues and cell lines. Further, SOX2OT was found to target miR-194-5p in a sequence-specific manner and negatively regulated the expression of miR-194-5p. Knockdown of SOX2OT inhibited cell proliferation, invasion and migration of GC cells through increasing miR-194-5p expression and suppressing EMT both in vitro and in vivo. This study demonstrated that the SOX2OT/miR-194-5p axis played a regulatory role in the proliferation and mobility of GC cells through suppressing EMT.

LncRNA SOX2OT is transcribed in the same orientation as SOX2 and regulated the transcription of SOX2 which was pivotal in tumorigenesis, acting as an enhancer (18). Accumulating evidence indicated that SOX2OT was strikingly elevated in various types of cancers and served as an oncogenic role. For instance, Liu found that SOX2OT was increased in colorectal cancer tissues and cell lines. Decreased SOX2OT expression inhibited cell proliferation, invasion, migration and EMT in colorectal cancer cells (11). Shi's study reported that SOX2OT expression level was significantly higher in hepatocellular carcinoma (HCC) tissues compared with adjacent non-tumor tissues. In addition, the metastasis ability of HCC cells was significantly restrained by knocking down lncRNA SOX2OT expression (18). As in GC, it was reported that SOX2OT overexpression served as a poor biomarker in GC (13). In accordance with these previous researches, in our present study, SOX2OT 

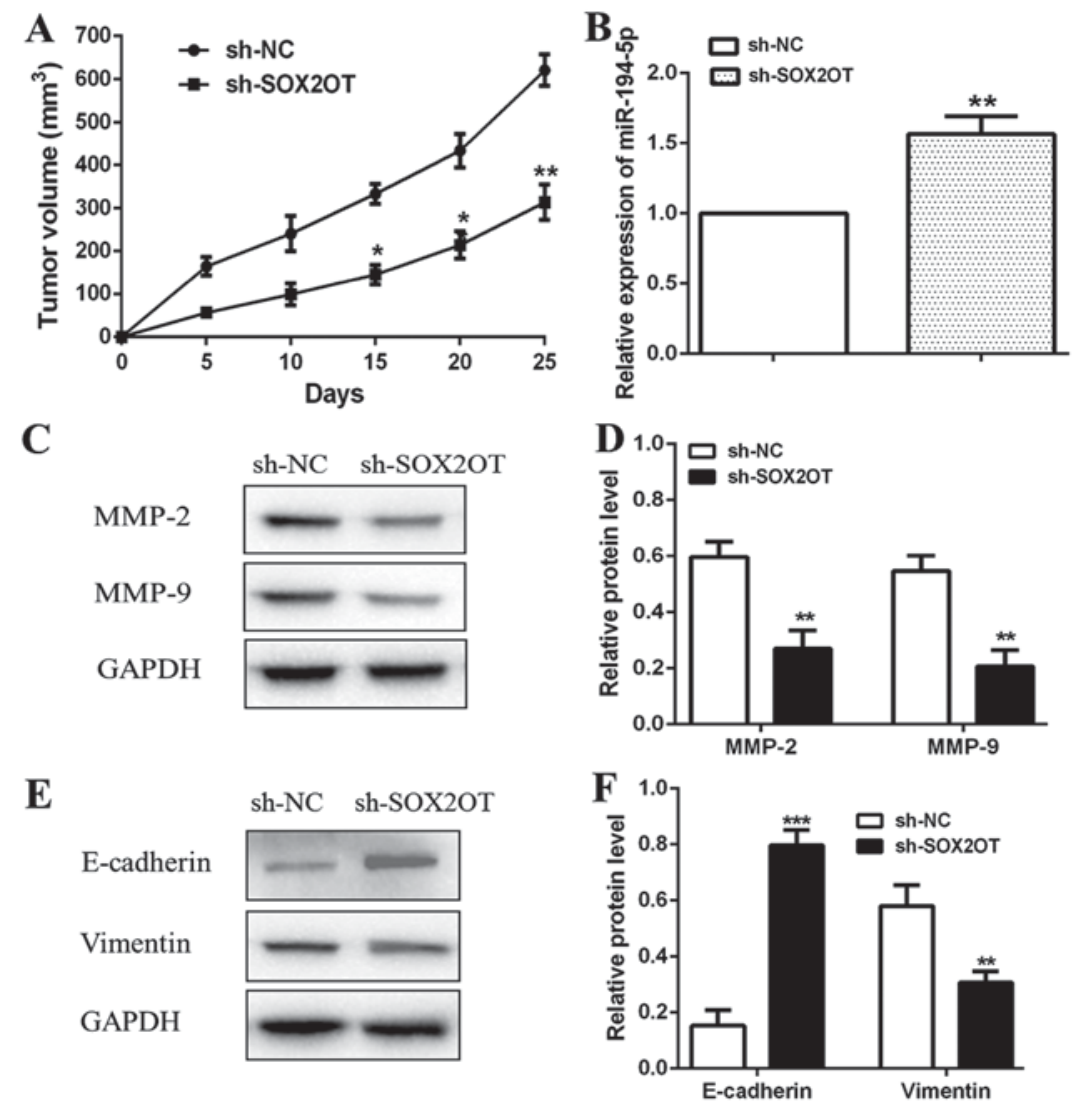

Figure 6. SOX2OT/miR-194-5p axis suppresses GC tumor growth and metastasis through inhibition of EMT in vivo. sh-NC or sh-SOX2OT transfected MKN-74 cells were injected into BALB/c nude mice, respectively. (A) Tumor volumes were measured every 5 days. (B) Relative expression of miR-194-5p was detected through RT-qPCR. (C) Representative western blot and (D) quantification of MMP-2 and MMP-9. (E) Representative western blot and (F) quantification of E-cadherin and Vimentin. All data are presented as the mean \pm standard deviation from three independent experiments. $\mathrm{P}<0.05$, ${ }^{* *} \mathrm{P}<0.01$ compared with sh-NC group. SOX2OT, SOX2 overlapping transcript; miR, microRNA; GC, gastric cancer; EMT, epithelial-mesenchymal transition; RT-qPCR, reverse transcription-quantitative polymerase chain reaction; MMP, matrix metalloproteinase.

expression was also found highly up-regulated in GC tissues and cell lines. In addition, knockdown of SOX2OT remarkably impeded cell proliferation, invasion and migration in GC cells, suggesting the oncogenic role of SOX2OT in the progression of GC. EMT is a cellular process that epithelial features are lost and mesenchymal features gradually develop. A large body of studies determined that EMT can reduce intercellular adhesion and promote cells metastasis in many types of cancer cells (19). During EMT, epithelial marker E-cadherin is down-regulated while the mesenchymal markers $\mathrm{N}$-cadherin and Vimentin are up-regulated in tumor cells (20). Matrix metalloproteinase (MMP) family members including MMP-2 and MMP-9 which can degrade extracellular matrix to promote metastasis of cells were also up-regulated during EMT (21). In our study, we observed that knockdown of SOX2OT increased E-cadherin expression while decreased $\mathrm{N}$-cadherin and Vimentin expression notably, indicating that knockdown of SOX2OT reduced EMT in GC cells. In summary, results above elucidated that knockdown of SOX2OT inhibited cell proliferation and mobility through suppressing EMT in GC cells.

Since we had revealed the role of SOX2OT in the progression of $\mathrm{GC}$, then we further investigated the underlying mechanism in detail. Previous researches demonstrated that LncRNAs could bind to common miRNA binding sites of mRNAs and abolished the downstream effects of these miRNA (22). Su reported that knockdown of SOX2OT could inhibit the malignant biological behaviors of glioblastoma stem cells via up-regulating the expression of miR-194-5p and miR-122 (16). Here, we also focus on the relationship between SOX2OT and miR-194-5p in GC. miR-194-5p expression was suppressed in glioblastoma multiforme (GBM) and miR-194-5p hampered cell growth and promoted apoptosis of GBM cells (23). Overexpression of miR-194-5p increased E-cadherin expression and inhibited invasion and migration of colorectal cancer cells (24). These results indicated the tumor suppressive role of miR-194-5p. In consistent with these previous studies, we also observed decreased miR-194-5p expression in GC tissues and cell lines. Further, through bioinformatics analysis, we found that SOX2OT might harbor a binding site for miR-194-5p. To verify this prediction, dual-luciferase reporter assay was performed, which demonstrated that SOX2OT could bind to miR-194-5p directly. Moreover, knockdown of SOX2OT increased the expression of miR-194-5p, suggesting that miR-194-5p was negatively regulated by SOX2OT in GC. Besides that, miR-194-5p inhibitor was found to counteract the inhibitory effects of SOX2OT on cell proliferation and mobility through enhancing EMT in GC cells. Taken together, our in vitro experiments revealed that knockdown of SOX2OT restrained cell proliferation and mobility through suppressing miR-194-5p and EMT in GC.

HavingunderstoodtheregulatingroleofSOX2OT/miR-194-5p axis in GC cells in vitro, we then carried out in vivo experiments for further investigation. Su's study revealed that sh-SOX2OT 
reduced tumor volume of glioblastoma xenograft model (16). Our results went in line with his study that knockdown of SOX2OT inhibited tumor growth and metastasis of GC through suppressing miR-194-5p and EMT in vivo.

In summary, our present study elucidated that SOX2OT was up-regulated in GC tissues and cell lines. Knockdown of SOX2OT inhibited cell proliferation and mobility of GC cells through increasing miR-194-5p expression. The SOX2OT/miR-194-5p axis participated in the tumor progression of GC through regulation of EMT both in vitro and in vivo. Hence, targeting the SOX2OT/miR-194-5p axis can be instrumental in establishing new strategies for GC therapy.

\section{Acknowledgements}

Not applicable.

\section{Funding}

No funding was received.

\section{Availability of data and materials}

The datasets used and/or analyzed during the current study are available from the corresponding author on reasonable request.

\section{Authors' contributions}

RQW designed the study and wrote the manuscript. CD, RAR and MDMRM performed the experiments, and reviewed and edited the manuscript. All authors read and approved the manuscript.

\section{Ethics approval and consent to participate}

The study was approved by the Medical Ethics Committee of Universidad de Almería. Informed consent was obtained from all individual participants included in the study. Animal experiments with mice were conducted strictly in accordance with a protocol approved by the Administrate Panel on Laboratory Animal Care of China Medical University and the animal experiments were approved by the Medical Ethics Committee of Universidad de Almería.

\section{Patient consent for publication}

Written informed consent was obtained from all patients for the publication of their data.

\section{Competing interests}

The authors declare that they have no competing interests.

\section{References}

1. Marqués-Lespier JM1, González-Pons M and Cruz-Correa M: Current perspectives on gastric cancer. Gastroenterol Clin North Am 45: 413-428, 2016.

2. Siegel RL, Miller KD and Jemal A: Cancer statistics, 2015. CA Cancer J Clin 65: 5-29, 2015.
3. Zhong J, Chen Y and Wang LJ: Emerging molecular basis of hematogenous metastasis in gastric cancer. World J Gastroenterol 22: 2434-2440, 2016.

4. Guttman M and Rinn JL: Modular regulatory principles of large non-coding RNAs. Nature 482: 339-346, 2012

5. Yan H, Yuan J, Gao L, Rao J and Hu J: Long noncoding RNA MEG3 activation of p53 mediates ischemic neuronal death in stroke. Neuroscience 337: 191-199, 2016.

6. Liu X, Hou L, Huang W, Gao Y, Lv X and Tang J: The mechanism of long non-coding RNA MEG3 for neurons apoptosis caused by hypoxia: Mediated by miR-181b-12/15-LOX signaling pathway. Front Cell Neurosci 10: 201, 2016.

7. Kam Y, Rubinstein A, Naik S, Djavsarov I, Halle D, Ariel I, Gure AO, Stojadinovic A, Pan H, Tsivin V, et al: Detection of a long non-coding RNA (CCAT1) in living cells and human adenocarcinoma of colon tissues using FIT-PNA molecular beacons. Cancer Lett 352: 90-96, 2014.

8. Avilion AA, Nicolis SK, Pevny LH, Perez L, Vivian N and Lovell-Badge R: Multipotent cell lineages in early mouse development depend on SOX2 function. Genes Dev 17: 126-140, 2003.

9. Fong H, Hohenstein KA and Donovan PJ: Regulation of self-renewal and pluripotency by Sox 2 in human embryonic stem cells. Stem Cells 26: 1931-1938, 2008.

10. Shahryari A, Jazi MS, Samaei NM and Mowla SJ: Long non-coding RNA SOX2OT: Expression signature, splicing patterns, and emerging roles in pluripotency and tumorigenesis. Front Genet 6: $196,2015$.

11. Liu S, Xu B and Yan D: Enhanced expression of long non-coding RNA Sox2ot promoted cell proliferation and motility in colorectal cancer. Minerva Med 107: 279-286, 2016.

12. Hou Z, Zhao W, Zhou J, Shen L, Zhan P, Xu C, Chang C, Bi H, Zou J, Yao X, et al: A long noncoding RNA Sox2ot regulates lung cancer cell proliferation and is a prognostic indicator of poor survival. Int J Biochem Cell Biol 53: 380-388, 2014.

13. Zhang Y, Yang R, Lian J and Xu H: LncRNA Sox2ot overexpression serves as a poor prognostic biomarker in gastric cancer. Am J Transl Res 8: 5035-5043, 2016.

14. Meister G: miRNAs get an early start on translational silencing. Cell 131: 25-28, 2007.

15. Wang SH, Wu XC, Zhang MD, Weng MZ, Zhou D and Quan ZW: Long noncoding RNA H19 contributes to gallbladder cancer cell proliferation by modulated miR-194-5p targeting AKT2. Tumour Biol 37: 9721-9730, 2016.

16. Su R, Cao S, Ma J, Liu Y, Liu X, Zheng J, Chen J, Liu L, Cai H, Li Z, et al: Knockdown of SOX2OT inhibits the malignant biological behaviors of glioblastoma stem cells via up-regulating the expression of miR-194-5p and miR-122. Mol Cancer 16: 171, 2017.

17. Livak KJ and Schmittgen TD: Analysis of relative gene expression data using real-time quantitative PCR and the 2(-Delta Delta C(T)) method. Methods 25: 402-408, 2001.

18. Shi XM and Teng F: Up-regulation of long non-coding RNA Sox 20 t promotes hepatocellular carcinoma cell metastasis and correlates with poor prognosis. Int J Clin Exp Pathol 8: 4008-4014, 2015.

19. Huber MA, Beug $\mathrm{H}$ and Wirth T: Epithelial-mesenchymal transition: NF-kappaB takes center stage. Cell Cycle 3: 1477-1480, 2004.

20. Singh A and Settleman J: EMT, cancer stem cells and drug resistance: An emerging axis of evil in the war on cancer. Oncogene 29: 4741-4751, 2010.

21. Coussens LM, Fingleton B and Matrisian LM: Matrix metalloproteinase inhibitors and cancer: Trials and tribulations. Science 295: 2387-2392, 2002.

22. Zhou X, Yuan P, Liu Q and Liu Z: LncRNA MEG3 regulates imatinib resistance in chronic myeloid leukemia via suppressing MicroRNA-21. Biomol Ther (Seoul) 25: 490-496, 2017.

23. Zhang Z, Lei B, Wu H, Zhang $X$ and Zheng N: Tumor suppressive role of miR-194-5p in glioblastoma multiforme. Mol Med Rep 16: 9317-9322, 2017.

24. Zhang Q, Wei T, Shim K, Wright K, Xu K, Palka-Hamblin HL, Jurkevich $A$ and Khare S: Atypical role of sprouty in colorectal cancer: Sprouty repression inhibits epithelial-mesenchymal transition. Oncogene 35: 3151-3162, 2016.

This work is licensed under a Creative Commons Attribution-NonCommercial-NoDerivatives 4.0 International (CC BY-NC-ND 4.0) License. 